\title{
Adjunctive Low-frequency Repetitive Transcranial Magnetic Stimulation over the Right Dorsolateral Prefrontal Cortex in Patients with Treatment-resistant Obsessive-compulsive Disorder: A Randomized Controlled Trial
}

\author{
Ho-Jun Seo, Young-Eun Jung ${ }^{2}$, Hyun Kook Lim¹, Yoo-Hyun Um¹, Chang Uk Lee ${ }^{3}$, Jeong-Ho Chae ${ }^{3}$ \\ Departments of Psychiatry, 'St. Vincent Hospital, Suwon, ${ }^{3}$ Seoul St. Mary's Hospital, Seoul, College of Medicine, The Catholic University \\ of Korea, ${ }^{2}$ Department of Psychiatry, Jeju National University School of Medicine, Jeju, Korea
}

\begin{abstract}
Objective: The present study aimed to evaluate the efficacy of low frequency (LF) repetitive transcranial magnetic stimulation (rTMS) over the right dorsolateral prefrontal cortex (DLPFC) for the treatment of obsessive-compulsive disorder (OCD). Methods: Twenty-seven patients with treatment resistant OCD were randomly assigned to 3 week either active $(n=14)$ or sham ( $n=13)$ rTMS. The active rTMS parameters consisted of $1 \mathrm{~Hz}, 20$-minute trains (1,200 pulses/day) at 100\% of the resting motor threshold (MT). OCD symptoms, mood, and anxiety were assessed at baseline and every week throughout the treatment period. Results: A repeated-measures analysis of variance (ANOVA) was used to evaluate changes on the Yale-Brown Obsessive Compulsive Scale (YBOCS). Our results revealed a significant reduction in YBOCS scores in the active group compared with the sham group after 3 weeks. Similarly, a repeated-measures ANOVA revealed significant effect of time and time Xgroup interaction on scores on the Hamilton Depression Rating Scale and the Clinical Global Impression-Severity scale. There were no reports of any serious adverse effects following the active and sham rTMS treatments.

Conclusion: LF rTMS over the right DLPFC appeared to be superior to sham rTMS for relieving OCD symptoms and depression in patients with treatment-resistant OCD. Further trials with larger sample sizes should be conducted to confirm the present findings.
\end{abstract}

KEY WORDS: Transcranial magnetic stimulation; Obsessive-compulsive disorder; Prefrontal cortex.

\section{INTRODUCTION}

Obsessive-compulsive disorder (OCD) is a chronic and highly debilitating neuropsychiatric disorder with a lifetime prevalence of 2-3\%. ${ }^{1,2)}$ Ego dystonic symptoms of OCD cause significant distress to patients as well as their family. ${ }^{3)}$ The substantial impairments that OCD causes in terms of interpersonal and occupational functioning have led to considerable social costs as well as this disorder's reputation as among the most disabling psychiatric conditions. $^{4)}$

Current first-line treatment strategies for OCD include high doses of selective serotonin reuptake inhibitors (SSRIs)

\footnotetext{
Received: August 13, 2015 / Revised: September 9, 2015

Accepted: October 1, 2015

Address for correspondence: Jeong-Ho Chae, MD Department of Psychiatry, College of Medicine, The Catholic University of Korea, 222 Banpo-daero, Seocho-gu, Seoul 06591, Korea

Tel: +82-2-2258-6083, Fax: +82-2-594-3870

E-mail: alberto@catholic.ac.kr
}

or clomipramine in conjunction with cognitive-behavioral therapy. ${ }^{5)}$ However, pharmacological treatment regimens are typically broadened to include various psychotropic agents, particularly antipsychotics. ${ }^{6}$ Even with a diverse array of therapeutic options, approximately $30-60 \%$ of OCD patients show only partial responses to treatment or are unable to tolerate the side effects related to these medications. ${ }^{7)}$ Additionally, a substantial portion of patients experiences persistent symptoms that impair their global functioning. ${ }^{8)}$

Repetitive transcranial magnetic stimulation (rTMS) is a non-invasive technique that may be an alternative strategy to effectively treat patients with OCD. rTMS delivers electromagnetic pulses to selective areas of the cerebral cortex via the direct application of an insulated wire coil to the scalp. The stimulating coil passes a rapidly alternating electrical current that generates a briefly pulsed magnetic field and results in the depolarization of the conducting neuronal tissue located just under the coil. ${ }^{9)}$

The dorsolateral prefrontal cortex (DLPFC) is one of

(c) This is an Open-Access article distributed under the terms of the Creative Commons Attribution Non-Commercial License (http://creativecommons.org/licenses/by-nc/4.0) which permits unrestricted non-commercial use, distribution, and reproduction in any medium, provided the original work is properly cited. 
the most interesting rTMS sites for the treatment of OCD patients because the pathophysiology of OCD is related to hyperactivity in specific cortical-subcortical loops that include the orbitofrontal cortex, anterior cingulate cortex, caudate nucleus, and DLPFC. ${ }^{10)}$ The DLPFC may represent a starting point for the induction of remote stimulation in connected cortico-subcortical circuits. ${ }^{11)} \mathrm{Neu}$ roimaging studies have shown that untreated OCD patients exhibit hypermetabolism and hyperperfusion in the prefrontal cortex, ${ }^{12)}$ that there is a normalization of prefrontal cortical activity following the administration of medication or neurosurgical treatments, ${ }^{13)}$ and that rTMS has a direct influence on prefrontal cortical activity. ${ }^{14)}$

However, there have been only few studies that have applied rTMS stimulation over the DLPFC for the treatment of OCD patients and they have produced conflicting results. ${ }^{15-22)}$ It is important to note that these studies employed heterogeneous designs in terms of stimulation site (right vs. left side), stimulus parameters (low frequency $[\mathrm{LF}]$ vs. high frequency $[\mathrm{HF}]$ ), session frequency and duration, sham conditions, and coil shape. Moreover, each of these studies used a small sample. The data from these studies are insufficient to provide conclusive results regarding the optimum protocol for the treatment of OCD patients.

The present study aimed to evaluate the efficacy of LF rTMS over the right DLPFC for the treatment of OCD patients. Although there is no consensus on side and frequency to stimulate DLPFC for OCD, several studies reported that metabolic rates was elevated in the right prefrontal cortex at resting state and treatment produced primarily right-sided changes in cerebral activity. ${ }^{16,23-25)}$ Thus, it was hypothesized that the administration of LF rTMS on the right DLPFC over 3 weeks, as an adjunctive treatment to pharmacotherapy, would improve the symptoms of patients with treatment-resistant OCD when compared with sham treatment.

\section{METHODS}

\section{Subjects}

The present study recruited patients with a primary diagnosis of OCD according to the criteria of the Diagnostic and Statistical Manual of Mental Disorders, 4th edition, text revision (DSM-IV-TR). The inclusion criteria for this study required that the subjects be right-handed, 18-60 years of age, and had experienced residual OCD symptoms of at least a moderate severity according to the Yale-Brown Obsessive Compulsive Scale (YBOCS; score
216) despite treatment with two anti-OCD medications. ${ }^{21)}$ Subjects with comorbid psychiatric disorders other than depression; a history of epilepsy, drug abuse, significant head injury, or any neurosurgical procedure; metal implants; and pacemakers as well as those who had received electroconvulsive therapy in the past 6 months were excluded from the present study. Written informed consent was obtained from all patients prior to participation in the study, and the study protocol was approved by the Institutional Review Board of St. Mary's Hospital in Seoul, Korea (SCMC05EA078).

\section{Procedure}

The patients were randomly assigned to receive either a sham procedure or active LF rTMS based on a computer-generated randomization schedule. The rTMS procedure was conducted using a TAMAS stimulator with a figure-eight coil (REMED, Daejeon, Korea; http://www. remed.kr). The stimulation parameters were performed for 3 weeks as follows: $1 \mathrm{~Hz}, 20$-minute trains $(1,200$ pulses/day) at $100 \%$ of the resting motor threshold (MT) once per day 5 days per week. The resting MT was determined using the thumb-movement visualization method via the stimulation of the primary motor cortex area for the left abductor pollicis brevis (APB) muscle. The stimulation site for the rTMS was over the right DLPFC, which was identified by measuring $5 \mathrm{~cm}$ anterior to and in a parasagittal line from the point of the maximum stimulation of the contralateral APB muscle. The sham rTMS procedure was identical to that of the active rTMS, except that a sham coil was used.

Since the rTMS sessions in the present study were performed as adjunctive therapies to ongoing medication regimens, the pharmacotherapies were continued in same

Table 1. Demographic and clinical characteristics of the subjects

\begin{tabular}{|c|c|c|}
\hline Variable & $\begin{array}{l}\text { Active rTMS } \\
\quad(n=14)\end{array}$ & $\begin{array}{c}\text { Sham rTMS } \\
(n=13)\end{array}$ \\
\hline Age $(y r)$ & $34.6 \pm 9.8$ & $36.3 \pm 12.5$ \\
\hline Gender (male/female) & $8 / 6$ & $6 / 7$ \\
\hline Marital status (married) & $8(57.1)$ & $7(53.8)$ \\
\hline Duration of illness (yr) & $9.8 \pm 8.0$ & $11.7 \pm 9.5$ \\
\hline Comorbid MDD & $12(85.7)$ & $10(76.9)$ \\
\hline \multicolumn{3}{|l|}{ Medication in use } \\
\hline Antidepressant & $14(100)$ & $13(100)$ \\
\hline Antipsychotics & $8(57.1)$ & $9(69.2)$ \\
\hline Mood stabilizer & $1(7.1)$ & $0(0)$ \\
\hline Benzodiazepine & $8(57.1)$ & $7(53.8)$ \\
\hline
\end{tabular}

Values are presented as mean \pm standard deviation, number only, or number (\%).

rTMS, repetitive transcranial magnetic stimulation; MDD, major depressive disorder. 
dosages for the whole duration of the study (Table 1). The patients and the rater were blind to the group assignments but, for technical reasons, the clinicians who administered the rTMS were not blind. The OCD symptoms of the patients were assessed at baseline and every week during the treatment period. The YBOCS score ${ }^{26,27)}$ was the main outcome measure of the present study but additional information was gathered using the Clinical Global ImpressionSeverity (CGI-S) scale, ${ }^{28)}$ the seven-item Hamilton Depression Rating Scale (HAMD), ${ }^{29)}$ the Hamilton Anxiety Rating Scale (HAMA), ${ }^{30)}$ and the Beck Depression Inventory $(\mathrm{BDI}){ }^{31)}$

\section{Statistical Analysis}

The summary statistics are presented as means and standard deviations (SD) for continuous variables and as numbers and percentages for discrete variables. The demographic and clinical data for the active and sham groups were compared using $t$-tests, chi-square tests, or MannWhitney $U$-tests, as appropriate. A repeated-measures analysis of variance (ANOVA) using a mixed-effects model with a symmetric covariance structure was used to evaluate the group- and time-dependent effects of rTMS on the mean scores of the psychometric scales. Post hoc analysis was performed with the Bonferroni correction. Patients with a reduction $\geq 25 \%$ in their YBOCS score were classified as responders. A $p$ value $<0.05$ (twotailed) was considered to indicate statistical significance, and all statistical analyses were conducted using Statistical Analysis System, ver. 9.1 (SAS Institute, Cary, NC, USA).

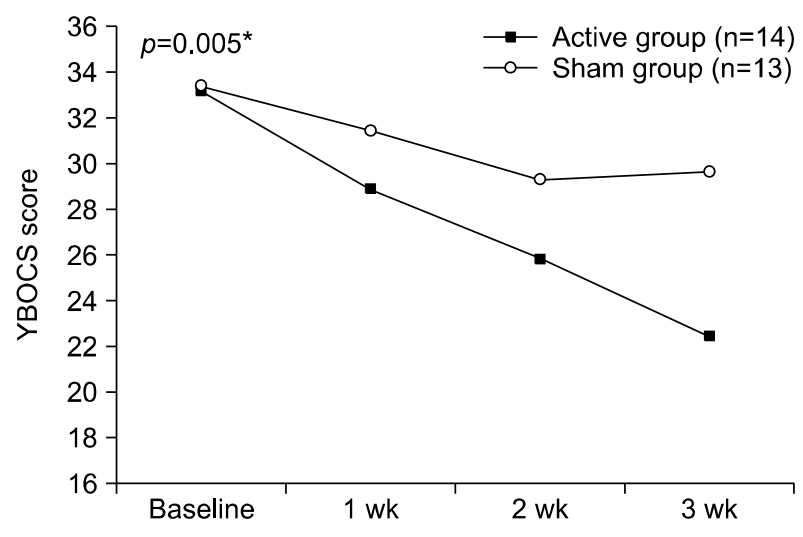

Fig. 1. Comparison of Yale-Brown Obsessive Compulsive Scale (YBOCS) scores in active and sham rTMS (repeated transcranial magnetic stimulation) groups.

*Repeated-measures ANOVA, groupxtime interaction.

\section{RESULTS}

Of the 28 patients initially recruited for the present study, one dropped out prior to the start of treatment due to follow-up loss. Therefore, the final sample included in the analyses consisted of 27 patients: 14 in the active group and 13 in the sham group. All patients completed at least $70 \%$ of all treatment sessions ( $>10$ sessions), and the procedure was well-tolerated. The active and sham groups did not significantly differ in terms of any clinical or demographic characteristics (Table 1).

Figure 1 illustrates the mean YBOCS scores over time throughout the treatment period. After 3 weeks of treatment, the patients in the active group had a mean reduction of 10.7 points (SD, 8.2 points), whereas the sham group had a mean reduction of 3.7 points (SD, 3.7 points). A repeated-measures ANOVA revealed a non-significant effect of group $(\mathrm{F}=0.328, p=0.572)$, a highly significant effect of time $(\mathrm{F}=22.502, p<0.001)$, and a significant time $\times$ group interaction $(\mathrm{F}=4.751, p=0.005)$. Post hoc analysis revealed a significant difference between the two groups at week $3(\mathrm{~F}=4.217, p=0.008)$.

Table 2 describes changes in the HAMD, HAMA, BDI, and CGI-S scores over time throughout the treatment period. Similar to the results for the YBOCS scores, a repeated-measures ANOVA analyzing the HAMD and CGI-S scores revealed a significant effect of time $(p<$ 0.001 and $p<0.001$, respectively) and a significant group $\times$ time interaction ( $p=0.028$ and $p=0.030$, respectively). Post hoc analysis revealed significant differences between the two groups in the HAMD at week 2 and in the CGI-S at week $3(\mathrm{~F}=2.557, p=0.009$ and $\mathrm{F}=2.900$, $p=0.008$, respectively).

However, the HAMA and BDI scores reflected only a significant effect of time $(p=0.001$ and $p=0.003$, respectively) with no significant group or group $\times$ time interaction effects.

An analysis of the percentage reduction in YBOCS scores after rTMS treatment showed that seven patients in the active group (50.0\%) and three patients in the sham group $(23.1 \%)$ were classified as responders $(p=0.148)$. Comparisons between responder and non-responder in the active group showed no significant differences in demographic characteristics, medication, and baseline scores of YBOCS, HAMD, HAMA, BDI, and CGI-S. There were no reports of any serious adverse effects following the rTMS or sham treatments, and the most common complaint following active treatment was localized scalp pain $(n=3)$; however, this did notpersist after the stimulation. 
Headache was reported by two patients in the active group, but this was resolved spontaneously within a few hours of treatment.

\section{DISCUSSION}

The present study investigated the effects of LF rTMS applied over the right DLPFC in patients with treatment-resistant OCD. The 3-week adjunctive rTMS treatment significantly improved the obsessive symptoms of the patients as well as their comorbid depressive symptoms.

The key component of OCD pathophysiology is linked to hyperactivity of the orbitofrontal-subcortical circuit. ${ }^{10,32)}$ It involves dysfunction in structures such as the orbitofrontal cortex, DLPFC, anterior cingulate cortex, and striatum. ${ }^{33,34)}$ Among these structures, the DLPFC is the most accessible site to rTMS stimulation owing to its superficial location. ${ }^{35)}$ The DLPFC is a higher-order brain region that is implicated in executive processes and cognitive control, including the ability to focus thought and flexibly shift that focus according to the environment. ${ }^{36}$ The DLPFC can be an important gateway for indirect effect on subcortical structure. ${ }^{33)}$ Two effective treatment strategies for OCD such as cognitive behavioral therapy and SSRIs led to a decrease in the hypermetabolism observed in the DLPFC. ${ }^{37)}$ The rTMS stimulation to the DLPFC could possibly normalize orbitofrontal cortex hyperactivity via activation of the inhibitory indirect pathway or via direct connections between the DLPFC and orbitofrontal cortex. ${ }^{38)}$ In healthy subjects, HF rTMS to the DLPFC was found to modulate dopamine release in orbitofrontal cortex and caudate nucleus, ${ }^{39,40)}$ whereas LF rTMS reduced regional blood floor in the orbitofrontal cortex. $^{41)}$

Neuroimaging studies of OCD have not provided consistent data which suggest hemisphere-dependent functional difference of the DLPFC. Functional magnetic resonance imaging (fMRI) studies generally reported that activation change was found in bilateral DLPFC during symptom provocation task. ${ }^{37,42)}$ Nevertheless, there were also several studies implicating hemispheric asymmetries in OCD. ${ }^{23,24,43)}$ These studies suggested predominantly right-sided changes in cerebral activity correlated with treatment response. A recent rTMS study reported that the stimulation to the bilateral pre-SMA increased right hemisphere resting MT, thereby normalizing baseline motor cortex hyper-excitability. ${ }^{44)}$ On the other hand, the application of rTMS over the DLPFC suggests a rather strong laterality effect. LF rTMS applied over the right DLPFC 
affects the same parts of cortico-subcortical circuits as does HF rTMS applied over the left side DLPFC. ${ }^{45,46)}$ The laterality effect of prefrontal rTMS is prominent in patients with depression and also observed in healthy subjects and patients with mania. ${ }^{14,47,48)}$ The neurophysiological mechanism of rTMS, particularly the role of stimulation frequency and laterality remain unclear. However, rTMS applied over the DLPFC induced cerebral blood floor changes which were not restricted to the stimulated site, but involved both sides of cortico-subcortical circuits including the bilateral orbitofrontal cortex. $^{41)}$ In this regard, our findings suggest that the right DLPFC might be one of the promising loci of rTMS stimulation for modulating orbitofrontal-subcortical circuit in OCD. The laterality-frequency interaction of the prefrontal rTMS will continue to be an ongoing research challenge in OCD.

Previous open trials of rTMS on OCD have shown that stimulation over the DLPFC is effective to reduce OCD symptoms. ${ }^{15,20)}$ Of the nine randomized sham-controlled trials that have been conducted to date, five applied rTMS to the prefrontal cortex and reported conflicting results regarding the amelioration of OCD symptoms (Table 3). ${ }^{17-19,21,22)}$ These studies found that rTMS either resulted in a significant reduction in the YBOCS scores of both the active and sham groups without producing statistically significant differences between the treatment groups at the study endpoints ${ }^{17-19)}$ or does not result in any therapeutic efficacy for treatment-resistant OCD subjects in either treatment group. $^{21,22)}$ These studies, which all focused on the DLPFC, generally showed that the active rTMS condition was not superior to the sham rTMS condition; however, there are several reasons that these findings may have been inconclusive. For example, the small sample sizes of the studies restrict the generalizability of the results and, moreover, these studies employed a variety of stimulation parameters, application sites, and numbers of sessions. The short treatment durations (generally 2 weeks) may have also been related to the negative results, as previous studies have shown that the main determinants of the efficacy of rTMS include intensity, number of pulses, and number of sessions. ${ }^{49)}$

Of these studies, Alonso et al. ${ }^{22)}$ adopted an approach that was similar to that of the present study, in which LF stimulation was applied over the right DLPFC. The reasons for the discrepancies between the findings of these two studies are unclear but, despite the similarity of the stimulus parameters and application site, several differences in terms of the technical points of these two studies

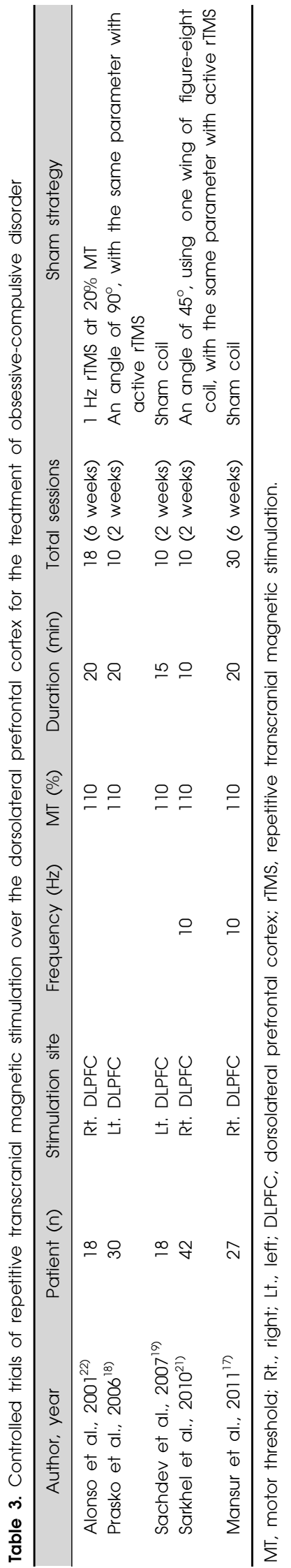


should be noted. First, Alonso et al. ${ }^{22)}$ employed a circular coil, which tends to induce a less focal current than the current induced by a figure- eight shaped coil. ${ }^{50)}$ Second, the sham condition in the Alonso study ${ }^{22)}$ was performed using $1 \mathrm{~Hz}$ rTMS at 20\% MT, which may have been active and could have interfered with the results from the placebo group. Third, these authors administered rTMS three times per week for 6 weeks and, despite the similar number of total sessions, the longer intervals between rTMS sessions may have influenced the treatment outcomes. ${ }^{16)}$

The present study had several limitations. First, the small sample size may restrict the generalizability of the results. Second, the lack of a double-blind procedure may have also confounded the results obtained in the placebo group. Third, the DLPFC was located using the " $5 \mathrm{~cm}$ rule, ${ }^{, 51)}$ which does not consider the shape and size of a patients' head. The use of a MRI-based neuro-navigation system would have improved the precision of the rTMS application. Fourth, the lack of a long-term follow-up period resulted in the absence of information regarding the maintenance of treatment gains following the rTMS procedure. Fifth, there would be considerable variability in neurophysiological response to rTMS among subjects in the current study. ${ }^{52)}$ Besides the genetic factor which decides intrinsic neuronal property, ${ }^{53)}$ medications such as benzodiazepine could also affect the threshold for rTMS by inducing changes in cortical excitability. ${ }^{54)}$ Especially, a long-term use of benzodiazepine was found to be associated with increased motor excitability threshold. ${ }^{54}$ Although no difference was found in current benzodiazepine usage between active and sham group and between responders and non-responders among active rTMS group, we cannot rule out the possibility of undetectable medication effects considering long illness duration of the patients in the current study. Finally, previous studies have suggested that the reduction in YBOCS scores may not be specific to the anti-obsessional effects induced by rTMS, but that they are related to non-specific antidepressant effects. $^{18,19)}$

Despite these limitations, the present findings suggest that LF rTMS applied over the right DLPFC may be a stimulation protocol that effectively treats patients with OCD. Further studies should be conducted to determine the optimal stimulation characteristics for the delivery of rTMS.

\section{Acknowledgments}

This study was supported by a grant from Seoul R\&BD Program (SS110008). The mechanics and the hardware were supported by CR Tech (now REMED Inc.), Daejeon, Republic of Korea.

\section{REFERENCES}

1. Karno M, Golding JM, Sorenson SB, Burnam MA. The epidemiology of obsessive-compulsive disorder in five US communities. Arch Gen Psychiatry 1988;45:1094-1099.

2. Weissman MM, Bland RC, Canino GJ, Greenwald S, Hwu $\mathrm{HG}$, Lee CK, et al. The cross national epidemiology of obsessive compulsive disorder. The Cross National Collaborative Group. J Clin Psychiatry 1994;55 Suppl:5-10.

3. Abramowitz JS, Taylor S, McKay D. Obsessive-compulsive disorder. Lancet 2009;374:491-499.

4. DuPont RL, Rice DP, Shiraki S, Rowland CR. Economic costs of obsessive-compulsive disorder. Med Interface 1995;8:102-109.

5. Stein DJ, Koen N, Fineberg N, Fontenelle LF, Matsunaga $\mathrm{H}$, Osser D, et al. A 2012 evidence-based algorithm for the pharmacotherapy for obsessive-compulsive disorder. Curr Psychiatry Rep 2012;14:211-219.

6. Abudy A, Juven-Wetzler A, Zohar J. Pharmacological management of treatment-resistant obsessive-compulsive disorder. CNS Drugs 2011;25:585-596.

7. Simpson HB, Huppert JD, Petkova E, Foa EB, Liebowitz $\mathrm{MR}$. Response versus remission in obsessive-compulsive disorder. J Clin Psychiatry 2006;67:269-276.

8. Pallanti S, Hollander E, Bienstock C, Koran L, Leckman J, Marazziti D, et al; International Treatment Refractory OCD Consortium. Treatment non-response in OCD: methodological issues and operational definitions. Int J Neuropsychopharmacol 2002;5:181-191.

9. George MS, Nahas Z, Kozel FA, Li X, Denslow S, Yamanaka K, et al. Mechanisms and state of the art of transcranial magnetic stimulation. J ECT 2002;18:170-181.

10. Aouizerate B, Guehl D, Cuny E, Rougier A, Bioulac B, Tignol J, et al. Pathophysiology of obsessive-compulsive disorder: a necessary link between phenomenology, neuropsychology, imagery and physiology. Prog Neurobiol 2004; 72:195-221.

11. Teneback CC, Nahas Z, Speer AM, Molloy M, Stallings LE, Spicer $\mathrm{KM}$, et al. Changes in prefrontal cortex and paralimbic activity in depression following two weeks of daily left prefrontal TMS. J Neuropsychiatry Clin Neurosci 1999; 11:426-435.

12. Rauch SL, Jenike MA, Alpert NM, Baer L, Breiter HC, Savage CR, et al. Regional cerebral blood flow measured during symptom provocation in obsessive-compulsive disorder using oxygen 15-labeled carbon dioxide and positron emission tomography. Arch Gen Psychiatry 1994; 51:62-70.

13. Hoehn-Saric R, Benkelfat C. Structural and functional brain imaging in obsessive compulsive disorder. In: Hollander E, Zohar J, Marazzati D, editors. Current insights in obsessive compulsive disorder. Chichester, NY:John Wiley \& Sons; 1994. p.183-211.

14. George MS, Wassermann EM, Williams WA, Steppel J, Pascual-Leone A, Basser P, et al. Changes in mood and hormone levels after rapid-rate transcranial magnetic stimulation (rTMS) of the prefrontal cortex. J Neuropsychiatry Clin Neurosci 1996;8:172-180.

15. Greenberg BD, George MS, Martin JD, Benjamin J, Schlaepfer TE, Altemus $\mathrm{M}$, et al. Effect of prefrontal repetitive transcranial magnetic stimulation in obsessive- 
compulsive disorder: a preliminary study. Am J Psychiatry 1997; 154:867-869.

16. Jaafari N, Rachid F, Rotge JY, Polosan M, El-Hage W, Belin D, et al. Safety and efficacy of repetitive transcranial magnetic stimulation in the treatment of obsessive-compulsive disorder: a review. World J Biol Psychiatry 2012;13:164-177.

17. Mansur CG, Myczkowki ML, de Barros Cabral S, Sartorelli Mdo C, Bellini BB, Dias AM, et al. Placebo effect after prefrontal magnetic stimulation in the treatment of resistant obsessive-compulsive disorder: a randomized controlled trial. Int J Neuropsychopharmacol 2011;14:1389-1397.

18. Prasko J, Pasková B, Záleský R, Novák T, Kopecek M, Bares $\mathrm{M}$, et al. The effect of repetitive transcranial magnetic stimulation (rTMS) on symptoms in obsessive compulsive disorder. A randomized, double blind, sham controlled study. Neuro Endocrinol Lett 2006;27:327-332.

19. Sachdev PS, Loo CK, Mitchell PB, McFarquhar TF, Malhi GS. Repetitive transcranial magnetic stimulation for the treatment of obsessive compulsive disorder: a double-blind controlled investigation. Psychol Med 2007;37:1645-1649.

20. Sachdev PS, McBride R, Loo CK, Mitchell PB, Malhi GS, Croker VM. Right versus left prefrontal transcranial magnetic stimulation for obsessive-compulsive disorder: a preliminary investigation. J Clin Psychiatry 2001;62:981-984.

21. Sarkhel S, Sinha VK, Praharaj SK. Adjunctive highfrequency right prefrontal repetitive transcranial magnetic stimulation (rTMS) was not effective in obsessive-compulsive disorder but improved secondary depression. J Anxiety Disord 2010;24:535-539.

22. Alonso P, Pujol J, Cardoner N, Benlloch L, Deus J, Menchón JM, et al. Right prefrontal repetitive transcranial magnetic stimulation in obsessive-compulsive disorder: a double-blind, placebo-controlled study. Am J Psychiatry 2001;158:1143-1145.

23. Saxena S, Brody AL, Ho ML, Alborzian S, Maidment KM, Zohrabi N, et al. Differential cerebral metabolic changes with paroxetine treatment of obsessive-compulsive disorder vs major depression. Arch Gen Psychiatry 2002;59:250-261.

24. Kang DH, Kwon JS, Kim JJ, Youn T, Park HJ, Kim MS, et al. Brain glucose metabolic changes associated with neuropsychological improvements after 4 months of treatment in patients with obsessive-compulsive disorder. Acta Psychiatr Scand 2003;107:291-297.

25. Swedo SE, Schapiro MB, Grady CL, Cheslow DL, Leonard $\mathrm{HL}$, Kumar A, et al. Cerebral glucose metabolism in childhood-onset obsessive-compulsive disorder. Arch Gen Psychiatry 1989;46:518-523.

26. Goodman WK, Price LH, Rasmussen SA, Mazure C, Fleischmann RL, Hill CL, et al. The Yale-Brown Obsessive Compulsive Scale. I. Development, use, and reliability. Arch Gen Psychiatry 1989;46:1006-1011.

27. Goodman WK, Price LH, Rasmussen SA, Mazure C, Delgado P, Heninger GR, et al. The Yale-Brown Obsessive Compulsive Scale. II. Validity. Arch Gen Psychiatry 1989;46: 1012-1016.

28. Guy W. ECDEU assessment manual for psychopharmacology, revised. Rockville, MD:National Institute of Mental Health; 1976.

29. Hamilton M. A rating scale for depression. J Neurol Neurosurg Psychiatry 1960;23:56-62.

30. Hamilton M. The assessment of anxiety states by rating. $\mathrm{Br}$ $J$ Med Psychol 1959;32:50-55.

31. Beck AT, Steer RA, Brown GK. BDI-II. Beck depression inventory. 2nd ed. San Antonio, TX:Psychological Corporation; 1996.
32. Lapidus KA, Stern ER, Berlin HA, Goodman WK. Neuromodulation for obsessive-compulsive disorder. Neurotherapeutics 2014;11:485-495.

33. Baxter LR Jr, Schwartz JM, Mazziotta JC, Phelps ME, Pahl $\mathrm{JJ}$, Guze $\mathrm{BH}$, et al. Cerebral glucose metabolic rates in nondepressed patients with obsessive-compulsive disorder. Am J Psychiatry 1988; 145:1560-1563.

34. Nordahl TE, Benkelfat C, Semple WE, Gross M, King AC, Cohen RM. Cerebral glucose metabolic rates in obsessive compulsive disorder. Neuropsychopharmacology 1989;2:23-28.

35. Deng ZD, Lisanby SH, Peterchev AV. Electric field depth-focality tradeoff in transcranial magnetic stimulation: simulation comparison of 50 coil designs. Brain Stimul 2013;6:1-13.

36. Kunde W, Reuss H, Kiesel A. Consciousness and cognitive control. Adv Cogn Psychol 2012;8:9-18.

37. Nakao T, Nakagawa A, Yoshiura T, Nakatani E, Nabeyama $\mathrm{M}$, Yoshizato C, et al. Brain activation of patients with obsessive-compulsive disorder during neuropsychological and symptom provocation tasks before and after symptom improvement: a functional magnetic resonance imaging study. Biol Psychiatry 2005;57:901-910.

38. Bais M, Figee M, Denys D. Neuromodulation in obsessivecompulsive disorder. Psychiatr Clin North Am 2014;37:393-413.

39. Cho SS, Strafella AP. rTMS of the left dorsolateral prefrontal cortex modulates dopamine release in the ipsilateral anterior cingulate cortex and orbitofrontal cortex. PLoS One 2009;4:e6725.

40. Strafella AP, Paus T, Barrett J, Dagher A. Repetitive transcranial magnetic stimulation of the human prefrontal cortex induces dopamine release in the caudate nucleus. J Neurosci 2001;21:RC157.

41. Knoch D, Treyer V, Regard M, Müri RM, Buck A, Weber B. Lateralized and frequency-dependent effects of prefrontal rTMS on regional cerebral blood flow. Neuroimage 2006; 31:641-648.

42. Adler CM, McDonough-Ryan P, Sax KW, Holland SK, Arndt S, Strakowski SM. fMRI of neuronal activation with symptom provocation in unmedicated patients with obsessive compulsive disorder. J Psychiatr Res 2000;34:317-324.

43. Rauch SL, Dougherty DD, Malone D, Rezai A, Friehs G, Fischman AJ, et al. A functional neuroimaging investigation of deep brain stimulation in patients with obsessive-compulsive disorder. J Neurosurg 2006;104:558-565.

44. Mantovani A, Rossi S, Bassi BD, Simpson HB, Fallon BA, Lisanby SH. Modulation of motor cortex excitability in obsessive-compulsive disorder: an exploratory study on the relations of neurophysiology measures with clinical outcome. Psychiatry Res 2013;210:1026-1032.

45. George MS, Wassermann EM, Williams WA, Callahan A, Ketter TA, Basser $\mathrm{P}$, et al. Daily repetitive transcranial magnetic stimulation (rTMS) improves mood in depression. Neuroreport 1995;6:1853-1856.

46. Schutter DJ, van Honk J, d'Alfonso AA, Postma A, de Haan EH. Effects of slow rTMS at the right dorsolateral prefrontal cortex on EEG asymmetry and mood. Neuroreport 2001; 12:445-447.

47. Pascual-Leone A, Catalá MD, Pascual-Leone Pascual A. Lateralized effect of rapid-rate transcranial magnetic stimulation of the prefrontal cortex on mood. Neurology 1996; 46:499-502.

48. Grisaru N, Chudakov B, Yaroslavsky Y, Belmaker RH. Transcranial magnetic stimulation in mania: a controlled study. Am J Psychiatry 1998;155:1608-1610.

49. Gershon AA, Dannon PN, Grunhaus L. Transcranial magnetic 
stimulation in the treatment of depression. Am J Psychiatry 2003; 160:835-845.

50. Cohen LG, Roth BJ, Nilsson J, Dang N, Panizza M, Bandinelli S, et al. Effects of coil design on delivery of focal magnetic stimulation. Technical considerations. Electroencephalogr Clin Neurophysiol 1990;75:350-357.

51. George MS, Wassermann EM, Kimbrell TA, Little JT, Williams WE, Danielson AL, et al. Mood improvement following daily left prefrontal repetitive transcranial magnetic stimulation in patients with depression: a placebocontrolled crossover trial. Am J Psychiatry 1997;154:17521756.
52. Wassermann EM. Variation in the response to transcranial magnetic brain stimulation in the general population. Clin Neurophysiol 2002;113:1165-1171.

53. Singh R, Macdonell RA, Scheffer IE, Crossland KM, Berkovic SF. Epilepsy and paroxysmal movement disorders in families: evidence for shared mechanisms. Epileptic Disord 1999;1:93-99.

54. Palmieri MG, Iani C, Scalise A, Desiato MT, Loberti M, Telera S, et al. The effect of benzodiazepines and flumazenil on motor cortical excitability in the human brain. Brain Res 1999;815:192-199. 